\title{
MRCI-dependent scaling of the budding yeast DNA replication timing program
}

\author{
Amnon Koren, ${ }^{1}$ Ilya Soifer, and Naama Barkai ${ }^{2}$ \\ Department of Molecular Genetics, Weizmann Institute of Science, Rehovot 76100, Israel
}

\begin{abstract}
We describe the DNA replication timing programs of 14 yeast mutants with an extended $\mathrm{S}$ phase identified by a novel genome-wide screen. These mutants are associated with the DNA replication machinery, cell-cycle control, and dNTP synthesis and affect different parts of $S$ phase. In 13 of the mutants, origin activation time scales with the duration of $S$ phase. A limited number of origins become inactive in these strains, with inactive origins characterized by small replicons and distributed throughout $S$ phase. In sharp contrast, cells deleted of $M R C l$, a gene implicated in replication fork stabilization and in the replication checkpoint pathway, maintained wild-type firing times despite over twofold lengthening of $S$ phase. Numerous dormant origins were activated in this mutant. Our data suggest that most perturbations that lengthen $S$ phase affect the entire program of replication timing, rather than a specific subset of origins, maintaining the relative order of origin firing time and delaying firing with relative proportions. Mrcl emerges as a regulator of this robustness of the replication program.
\end{abstract}

[Supplemental material is available online at http://www.genome.org. The microarray data from this study have been submitted to the NCBI Gene Expression Omnibus (http://www.ncbi.nlm.nih.gov/geo) under accession no. GSE17120.]

Eukaryotic cells replicate their DNA in a highly orchestrated manner. DNA synthesis is initiated at specific chromosomal sites (replication origins) and proceeds in a bidirectional manner. Different loci are replicated at different times during $\mathrm{S}$ phase, depending on their distance from the nearest replication origin and on the time at which that origin initiates replication. This temporal order is highly reproducible between cells and can change concomitantly with cell differentiation (Hiratani et al. 2008; Schwaiger et al. 2009).

The process of DNA replication has been studied extensively. Replication origins are licensed during mitosis and early $G_{1}$, with the binding of ORC (origin recognition complex) and the subsequent recruitment of the minichromosome maintenance (MCM) complex (part of the replicative DNA helicase) (for review, see Bell and Dutta 2002; Aladjem 2007; Sclafani and Holzen 2007). The onset of $S$ phase is marked by the induction of the Clb5-6 cyclins (for review, see Murray 2004) and the associated degradation of the cyclin-dependent kinase (CDK) inhibitor Sic1 (Verma et al. 1997), leading to the phosphorylation of MCM subunits (via the cell cycle kinase Cdc7-Dbf4), and the recruitment of DNA polymerases $\alpha$ and $\varepsilon$ to licensed origins (via phosphorylated Sld2/ Sld3; Tanaka et al. 2007; Zegerman and Diffley 2007). Origins are then activated, with DNA polymerases catalyzing the addition of dNTPs to primer-template junctions.

Only little is known about the mechanism that regulates the timing at which specific origins are activated. Saccharomyces cerevisiae sic1 mutants, which enter S phase prematurely, replicate DNA from a smaller number of origins, presumably because of a failure to license a subset of origins (Lengronne and Schwob 2002). Late replication origins are inactive when the $S$ phase cyclin CLB5 is deleted (Donaldson et al. 1998) and when cells are subjected to DNA damage (by methyl methanesulfonate [MMS]) or

\footnotetext{
1Present address: Department of Genetics, Harvard Medical School, Boston, MA 02115, USA.

treated with hydroxyurea (HU), a drug that reversibly inhibits ribonucleotide reductase (RNR) required for reduction of nucleoside triphosphates (NTPs) to dNTPs. Notably, the replication checkpoint kinases Mec1 and Rad53 are required for this suppression (Santocanale and Diffley 1998; Shirahige et al. 1998). Recent work, however, demonstrated that rather than suppressing late origins, $\mathrm{HU}$ confers an overall slowdown of the replication program (Alvino et al. 2007).

To obtain further insight into the regulators of the replication program, we performed a genome-wide screen for mutants with an extended S phase. A total of 14 genes were identified, nine of which were not previously associated with $S$ phase duration. In 13 of the mutants, origin firing time appeared to scale with the duration of $\mathrm{S}$ phase. Scaling was lost, and numerous dormant origins were activated, in cells deleted for MRC1, a gene required for normal fork progression (Szyjka et al. 2005; Tourriere et al. 2005) and for transducing the checkpoint signal from Mec1 to Rad53 upon replication stress (Osborn and Elledge 2003).

\section{Results}

Identification of genes required for fast DNA replication

We screened systematically for nonessential genes that extended the duration of $S$ phase relative to that of the other cell-cycle phases, using an optimized DNA staining protocol that discriminates between $\mathrm{G}_{1^{-}}, \mathrm{S}$-, and $\mathrm{G}_{2}$-phase cells in an asynchronous culture (Fig. 1A, Methods). This assay reproduced the prolonged $\mathrm{S}$ phase of cells lacking the $S$ phase cyclin CLB5 (but not CLB6; Fig. 1B; Supplemental Fig. S1) (Donaldson et al. 1998), the replicationcheckpoint mediator MRC1, or the RRM3 helicase (Ivessa et al. 2003). Similarly, a shortened $G_{1}$ phase, followed by an extended $\mathrm{S}$ phase, was observed in cells deleted of the CDK inhibitor SIC1 (Lengronne and Schwob 2002) and cells lacking the SCF ubiquitin ligase DIA2 (Fig. 1B; Koepp et al. 2006; Morohashi et al. 2009).

We began by screening manually $\sim 200$ candidate genes known to be involved in DNA replication, cell cycle regulation, transcriptional control, chromatin structure, DNA repair and recombination, 


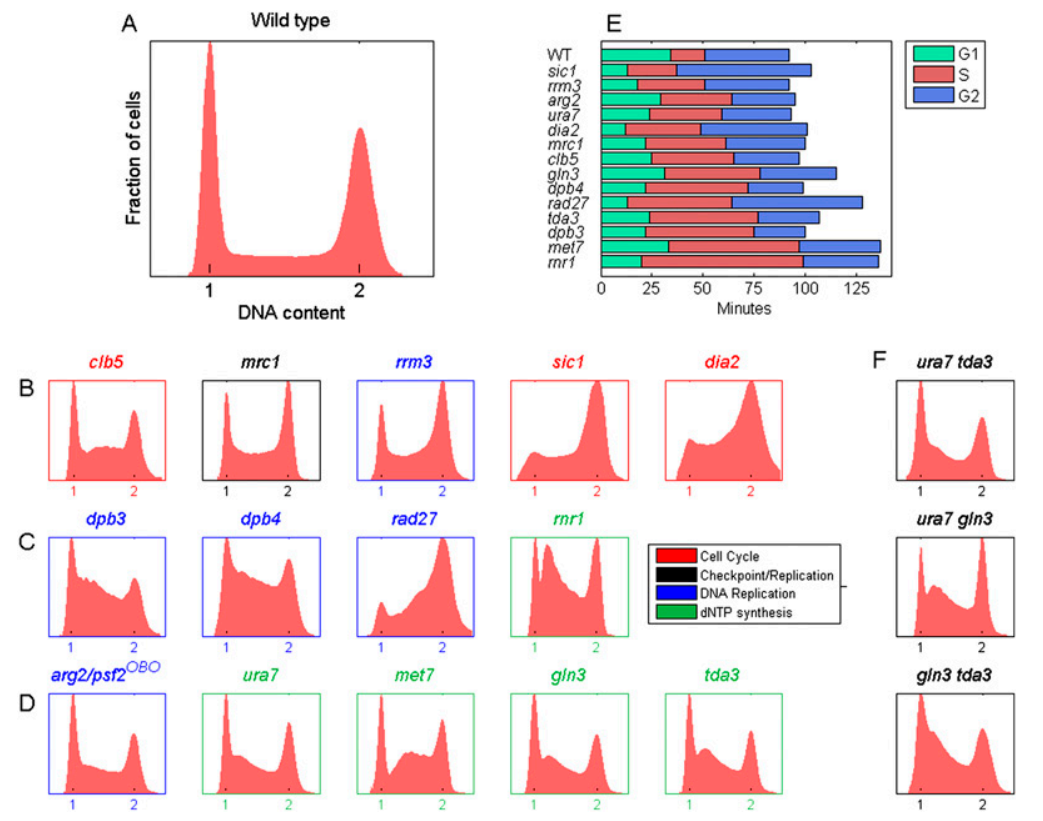

Figure 1. A screen for genes with extended $S$ phase. (A-D) Distribution of DNA content ("FACS profiles") in asynchronous populations of wild-type cells (BY4741) $(A)$ and cells identified in the screen $(B-D)$. All FACS profiles were reproduced at least eight times. The color codes of the titles and axes in $B-D$ denote known functional classifications of the genes. (OBO) Off-by-one (Pan et al. 2006). (E) Generation time and duration of each cell-cycle phase for the different mutants. Ordering is $S$ phase duration. See Supplemental Table $S 2$ for the raw data and summary of the genes and phenotypes. $(F)$ FACS profiles of double mutant combinations as indicated.

and chromosome segregation (Supplemental Table S1). Four S phase effectors were thus identified (Fig. 1C). Second, we used a robotic assay to screen the entire collection of viable yeast deletion mutants, obtaining data for $\sim 4500$ out of $\sim 4700$ library strains. This analysis reproduced all nine phenotypes defined manually and identified five additional effectors (Fig. 1D). S phase duration ranged from $\sim 17 \mathrm{~min}$ in wild-type cells to $79 \mathrm{~min}$ in the rnr1 mutant (Fig. 1E). Several of the mutants also had a concomitant shortening of the length of $\mathrm{G}_{1}$, including sic1 as previously reported. In contrast to previous suggestions, however (Lengronne and Schwob 2002), short $G_{1}$ per se did not lead necessarily to a longer S phase (see below and Supplemental Discussion 1). Several additional mutants displayed a marginal increase in $S$ phase duration and were not analyzed further (Supplemental Fig. S1). The limited number of genes identified may reflect the fact that our screen focused on nonessential genes while many DNA replication factors are expected to be essential. In addition, we found that many genes involved in various aspects of DNA replication, repair, and recombination display a normal S phase duration. We also note that in contrast to previous hypotheses (Knott et al. 2009 and references therein), our screen did not reveal a role for chromatin modifiers or transcriptional regulators in $\mathrm{S}$ phase duration.

The 14 genes identified belong to three functional groups. The first group ( $c l b 5$, sic1, dia2, and $m r c 1)$ is associated with cellcycle progression. The second group is associated with the DNA replication machinery and includes $D P B 3$ and $D P B 4$, the two nonessential subunits of DNA polymerase $\varepsilon$ (Araki et al. 1991; Ohya et al. 2000); RAD27, which encodes for the flap endonuclease 1 (FEN-1) enzyme required for removal of RNA primers from Okazaki fragments; and RRM3, which is required for the removal of nonhistone proteins that interfere with replication fork progression. This group may also include PSF2, an essential subunit of the GINS complex implicated in replication initiation and elongation (Sclafani and Holzen 2007). PSF2 is located in the genome adjacent to the $A R G 2$ gene identified in our screen, leading us to suspect that the effect of $\arg 2$ on $\mathrm{S}$ phase is due to it affecting PSF2 gene expression. Indeed, we found that the expression level of PSF2 decreases threefold in $\arg 2 \Delta$ relative to wild-type cells (data not shown).

The third group is associated with nucleotide metabolism. Genes in this group include $R N R 1$, a subunit of the RNR complex; URA7, a CTP synthetase (OzierKalogeropoulos et al. 1994) required for $\sim 90 \%$ of CTP synthesis; and MET7, a folylpolyglutamate synthetase required (indirectly) for dTMP synthesis (Cherest et al. 2000). We also identified GLN3, a transcription factor that controls nitrogen utilization genes (Blinder and Magasanik 1995), and TDA3 (YHROO9C), an uncharacterized yeast gene (Saccharomyces Genome Database, http://www.yeastgenome.org/).

\section{Distinct $\mathbf{S}$ phase profiles displayed by different mutants}

Examination of the FACS profiles (Fig. 1B-D) revealed that the mutants differ not only by the total length of $S$ phase but also by the distribution of cells within the $S$ phase itself. For example, $d b p 3$ and $d b p 4$ cells exhibited a specific lengthening of early S, whereas rad27 cells delayed DNA replication from mid $S$ to late $S$ phase. This later phenotype is consistent with a gradual increase in the number of Okazaki fragments as more origins are fired and as replication forks progress. Disruption of RNR1 generated a distinct intra-S peak, possibly indicating a replication block at a post-initiation step, followed by adaptation.

Interestingly, $t d a 3$ and $g \ln 3$ showed an extension of early $\mathrm{S}$ phase similarly to ura7, suggesting that they may be required for nucleotide (CTP) synthesis. Epistatic analysis further supported this possibility, as S phase profiles in ura $7 \mathrm{tda} 3$, ura $7 \mathrm{~g} \ln 3$, and $g \ln 3$ tda3 cells were virtually identical to the single-mutant profiles (Fig. 1F). Consistently, Tda3 has a predicted nucleotide binding domain (Saccharomyces Genome Database) and is localizes to the cytoplasm, implying an indirect effect on DNA replication. Gln3 is a transcription factor and may therefore regulate other gene(s) involved in a related pathway, although we have not identified any likely candidate.

\section{Epistatic interactions of $\mathrm{S}$ phase mutants}

Epistasis analysis provides a general means for defining the relationships between different genes associated with a particular phenotype. Focusing first on nucleotide metabolism (rnr1, met7, and $u$ ra7), we examined the effect of adding $\mathrm{HU}$, a drug that inhibits the catalytic subunit (Rnr2) of RNR and consequently reduces the levels of available nucleotides, and of deleting SML1 (RNR protein inhibitor) or RFX1 (RNR repressor) (Huang et al. 1998; Zhao et al. 1998, 2001). HU treatment of wild-type cells resulted in a dosedependent mid-S arrest (Fig. 2A). Addition of increasing HU doses

\section{Genome Research}


A
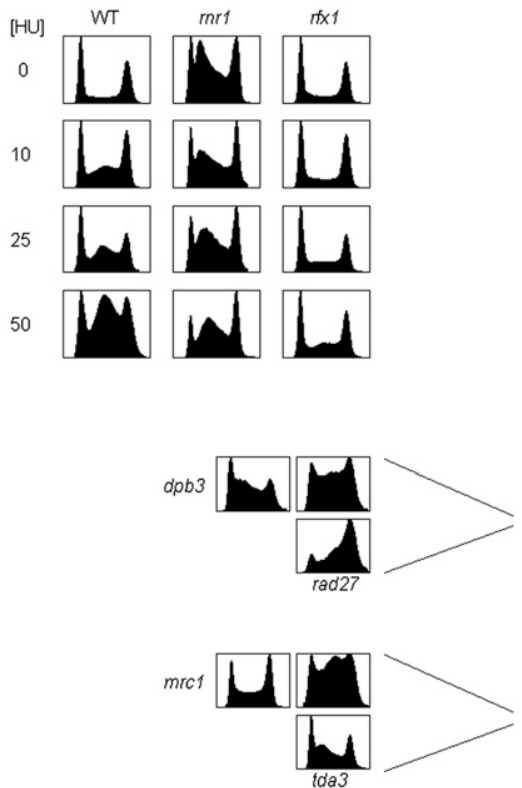

B
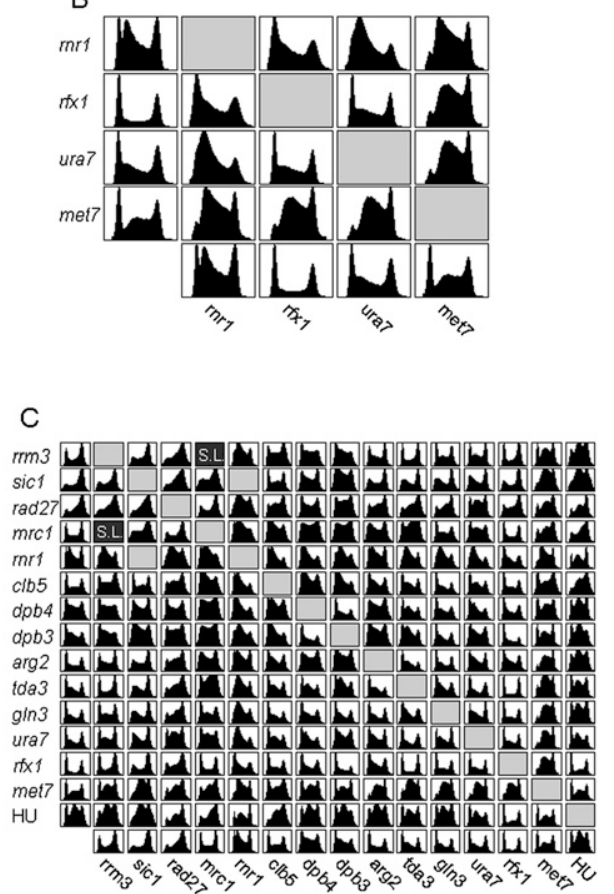

Figure 2. Genetic characterization of S-phase mutants. (A) FACS profiles of the indicated cells treated with different dosage of $\mathrm{HU}$. The large $\mathrm{G}_{2}$ fraction of HU-treated $r n r 1$ cells may be overestimated because of some difficulties in discriminating doublets (data not shown). (B) Synthetic interactions between genes associated with dNTP synthesis. FACS profiles are shown for mutants deleted of all genepair combinations. (C) A complete epistatic miniarray profile (E-Map) for the genes identified in our screen. Genetic interaction scores were computed and used to cluster the matrix (see Methods). (SL) Synthetic lethal. (Gray boxes) No data. Two cases that resulted in particularly long S phases are at left: $d p b 3 \mathrm{rad} 27$ and $\mathrm{mrc} 1 \mathrm{tda} 3$. Note the strong aggravating interaction in mid-to-late $\mathrm{S}$ phase not evident in either of the single mutants.

to $r n r 1$ cells converted the $S$ phase pattern to a pattern resembling $\mathrm{HU}$ treatment of wild-type cells. Notably, the HU phenotype was strongly suppressed by deletion of RFX1 (but not SML1) (Fig. 2A; Supplemental Fig. S1; Supplemental Discussion 2).

We generated strains deleted of all gene pair combinations including $r f \times 1$ (Fig. 2B). RFX1 deletion only partially suppressed the rnr1 phenotype, converting it to a pattern similar to that of ura 7 . This suggests that rnr 1 cells are not only deficient in the level of available nucleotides (a phenotype that is likely to be suppressed by $r f \times 1$ ) but are also limited in their ability to maintain proper balance between the different nucleotides. Indeed, Rnr1 contains allosteric sites for controlling both total dNTP levels as well as the balance between the four dNTPs (Nordlund and Reichard 2006). Surprisingly, aggravating interactions were observed between $r f x 1$ and $m e t 7$, as well as between met7 and ura7 (see also Supplemental Discussion 1, regarding the effect on $\mathrm{G}_{1}$ phase). Addition of $\mathrm{HU}$ to ura7 or met7 resulted in an alleviating interaction similar to that between $r n r 1$ and HU (Fig. 2C). Taken together, these phenotypes suggest that $\mathrm{S}$ phase duration depends not only on the availability but also on the balance between the different nucleotides.

We generated the full E-Map for 13 of the 14 genes identified (see Methods). As expected, DPB3 and DPB4 interacted in a highly similar fashion with most other genes, as did $u r a 7, g \ln 3$, and $t d a 3$, further supporting their assignment to the same pathway. Different genes had different tendencies for aggravating or alleviating interactions (Fig. 2C). For example, deletion of the checkpoint mediator $m r c 1$ further extended $\mathrm{S}$ phase delay of many mutants, suggesting that proper checkpoint function is required to buffer perturbations in multiple pathways. Overall, we could not identify clear grouping of genes, suggesting that the mutants represent parallel pathways and affect $S$ phase duration and DNA synthesis by distinct means.

\section{Characterization of the genomic DNA replication programs}

To gain further insights into the function of these mutants, we mapped their genome-wide replication program. Previous genome-wide analyses of replication timing in yeast relied on culture synchronization by mating pheromone and on artificial slowing of $\mathrm{S}$ phase (Raghuraman et al. 2001; Yabuki et al. 2002; Green et al. 2006; Alvino et al. 2007; McCune et al. 2008; Knott et al. 2009). We decided to follow an alternative approach, previously applied to Drosophila cells (Schubeler et al. 2002), in which the DNA content of FACSsorted $S$ phase population is analyzed. This measurement serves as a good proxy for DNA replication timing, since sequences replicated early are more abundant in asynchronous populations, whereas later replicating sequences are scarcer.

We FACS-sorted populations of $S$ and $\mathrm{G}_{1}$ cells, labeled them with different fluorescent dyes, and cohybridized them to an Agilent genomic tiling array (Fig. 3A). Each experiment was performed in four biological replicates. To control for data quality, we measured the correlation between the DNA content of adjacent chromosomal regions. As expected, this autocorrelation decayed gradually over a distance of $\sim 100 \mathrm{~kb}$, comparable to a typical replicon size, in contrast to a randomized data, where it disappeared practically immediately (Fig. 3C).

Replication profiles were obtained by plotting the (smoothed) data as a function of chromosomal coordinates (Fig. 3B). Peaks correspond to replication origins with their height reflecting the timing of origin activation. Valleys correspond to termini, and the replication rate of forks emanating from each origin is approximated by the adjacent slopes (Raghuraman et al. 2001). Our data were reproducible (Fig. 3B,E) and correlated well with previous reports: Peak locations corresponded to known positions of replication origins (Nieduszynski et al. 2006), with an average distance of $4.3 \mathrm{~kb}\left(P=4.47 \times 10^{-27}\right)$ (Fig. $\left.3 \mathrm{~B}\right)$, and origin activation timing and the overall profile were highly correlated with previous experiments (Fig. 3D,E; Supplemental Fig. S2).

We mapped the genome-wide replication timing profiles of all 14 mutants with extended S phase. Visual inspection indicated that for most strains, the replication pattern remains highly similar to that of wild type (Fig. 4A,B; Supplemental Fig. S3). Indeed, data from different mutants were highly correlated $(r=0.82-0.97$; mean $=$ 0.91 , compared with $r \sim 0.98$ between wild-type repeats; Fig. 4C). Particularly strong correlations were found between $d p b 3, d p b 4$, $\arg 2$ (psf2), and, to a somewhat lesser extent, clb5. Consistent with the genetic characterization above, $u r a 7, g \ln 3$, and $t d a 3$ formed an 

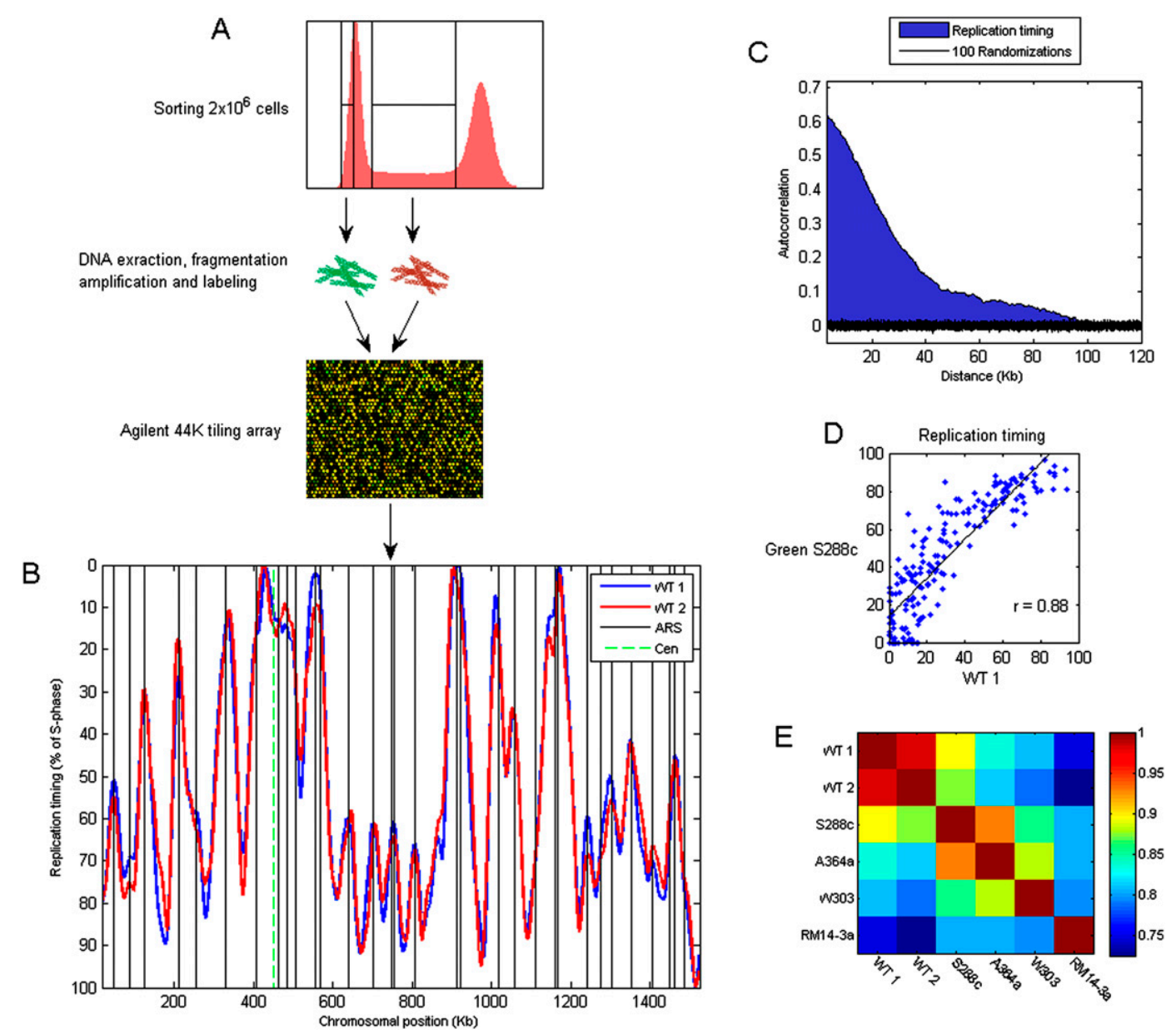

Figure 3. Profiling the genomic DNA replication timing program. (A) Two million SYBR-Green stained cells are sorted separately from $\mathrm{G}_{1}$ and $S$ phases, differentially labeled with fluorescent Cy dyes, and competitively hybridized to an Agilent genomic tiling array of $\sim 290$-bp resolution. The data from four biological replicates are averaged, and the averaged data are smoothed to generate the DNA replication timing profile. $(B)$ The replication profile of chromosome IV generated in two experiments, each consisting of four biological repetitions. The profiles are scaled to $0-100$, with 0 being the beginning of $S$ phase and 100 the end. The black vertical lines correspond to positions reported as replication origin ("verified" origins, identified by a combination of experimental and sequence analysis; Nieduszynski et al. 2006). (C) Autocorrelation as a quality control: Shown are the average correlations between the DNA content in chromosomal positions separated by the indicated distances. The correlations are calculated from the raw (unsmoothed) data, are averaged over all chromosomal positions, and are normalized to a maximum of 1 . The black region represents 100 separate calculations of autocorrelations on randomized data. $(D)$ Comparison of origin activation times in wild-type cells, as identified in our experiment, with those characterized previously using a similar strain background (S288c [Green et al. 2006]; our strain background is BY4741, a derivative of S288c). See Supplemental Figure S2 for comparison with other data sets. (E) A matrix showing the genome-wide correlations between different data set, including the two repeats from our study. The different experiments used different strain backgrounds, as indicated (S288c and A364a [Green et al. 2006]; W303 [Yabuki et al. 2002]; RM143a [Raghuraman et al. 2001]).

additional tight cluster with similarities comparable to those between wild-type repeats, making them practically indistinguishable. The one notable exception was the strain deleted of MRC1, whose replication profile was markedly different from the others (Fig. 4A,B), resulting in a significantly lower correlation with the rest of the strains $(r=0.56-0.79$; mean $=0.67$; Fig. $4 \mathrm{C})$.

The autocorrelation measure was also highly similar between 13 of the 14 strains, decaying more gradually than in wild type, suggesting a larger spacing between replication origins. Again, $m r c 1$ was distinctly different, displaying a sharp decrease in the autocorrelation values, indicative of more closely spaced origins (Fig. 4D).

\section{Scaling of the DNA replication program}

To examine the number of origins directly, we summarized the peak information from all replicate experiments into a set of 211 predicted origins (Fig. 5A, Methods), an estimate that is somewhat lower than previous reports because of our strict criteria. The number of predicted origins in $m r c 1$ cells was significantly higher, totaling 248 origins, and including 68 origins not active in wild type. Notably, these predicted origins were positioned at known origin sites (average distance $=5.5 \mathrm{~kb} ; P=7.2 \times 10^{-7}$ ), indicating that these are bona fide replication origins that are not detected in wild-type cells, possibly because of the faster rate of fork progression relative to mrc1 cells. The increase in the number of origins firing is consistent with previous reports showing activation of specific dormant origins in mrc1 mutants (Katou et al. 2003). All other mutants had fewer origins compared with wild type.

We compared systematically the timing of activation of each origin in the mutant vs. the wild-type strain (Fig. 5B,C; Supplemental Fig. S5). In most mutants, origin firing was significantly delayed but remained approximately scaled with the duration of 
A

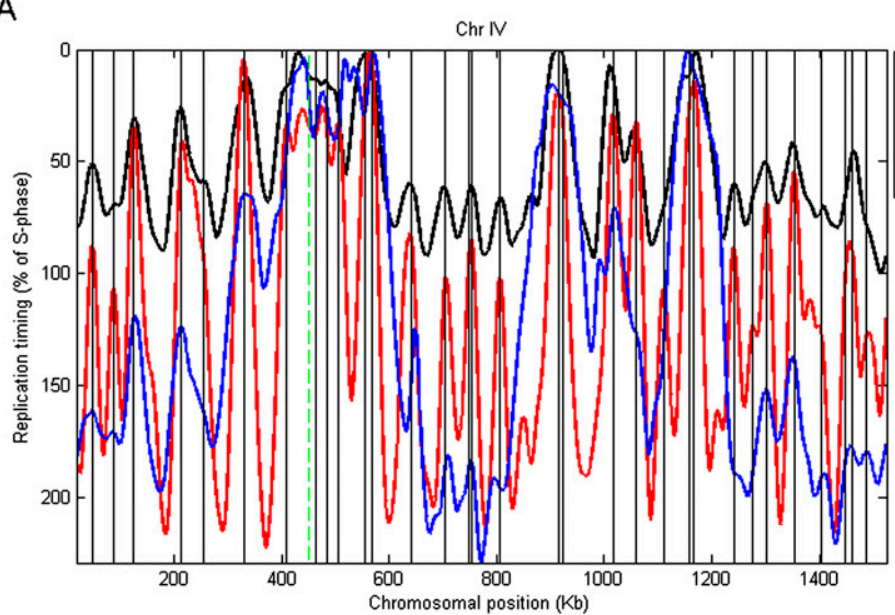

B
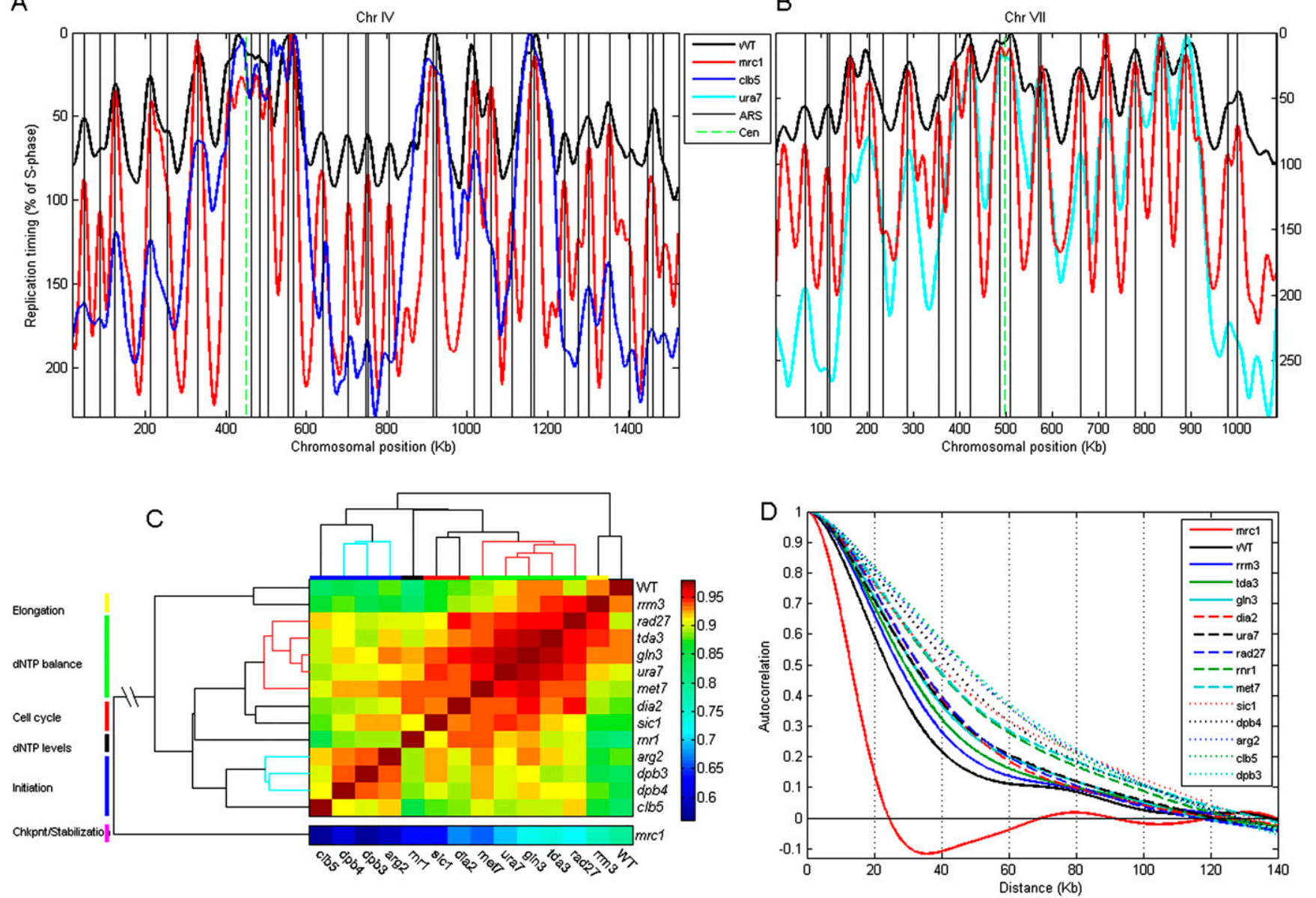

Figure 4. Replication timing profiles of the mutants identified in our screen. $(A, B)$ Replication profiles of chromosome IV $(A)$ and VII $(B)$ for wild-type, mrc1, and either $c l b 5(A)$ or ura7 (B). Axes, legends, and vertical lines are as in Fig. 3B, with the exception that the $y$-axis is scaled according to the length of $S$ phase in each of the mutants. Other mutants and chromosomes are shown in Supplemental Figure $S 3$. (C) Clustered correlation matrix of the entire data set. The dendrogram distance to $m r c 1$ is not to scale (broken lines). Functional classifications are based on the literature, the FACS results, and analysis of the microarray data described in the main text. Correlations were calculated between the smoothed averages of four biological repeats, as described above (Fig. 3). See Supplemental Figure S4 for correlation between individual repeats. (D) Autocorrelations (calculated as in Fig. 3C) of the different mutants: Strains are ordered by increasing values of autocorrelation at $50 \mathrm{~kb}$. Autocorrelation shown here was calculated using the smoothed profile. See Supplemental Figure $\$ 4$ for autocorrelations for the raw data.

$S$ phase. Thus, for example, origins activated in early-S in the wildtype cells were also activated early in the mutant backgrounds, whereas origins that fired during mid-S phase in wild type also fired in mid-S phase in the mutants. Several variations on this theme are worth noting: In $\mathrm{rrm} 3$ cells, the delay was confined to the last two-thirds of $S$ phase, whereas in the $r n r 1$ background origin delay leveled off and began to reverse toward the end of $S$ phase, reminiscent of the apparent recovery in S phase progression observed by FACS. Finally, in sic1, origins were delayed by a relatively constant magnitude throughout $S$ phase.

While the relative firing time of the different origins was maintained in the mutants and was generally consistent with the extension of S phase duration, scaling was not precise, and, in fact, delay of most origins was longer than expected for pure scaling and some origins became inactive (Fig. 5B; most origins reside above the black lines). Inactivated origins were characterized by small replicon size but were otherwise distributed throughout $S$ phase (Supplemental Figs. S5, S6; data not shown). Since replicon sizes naturally get smaller as S phase progresses (Supplemental Fig. S7), a larger fraction (but not number) of inactivated origins are late- replicating (Supplemental Fig. S6). This pattern is consistent with origins being inactivated because of interference, the passive replication by forks from adjacent origins reaching them before they have a chance to fire, rather than invoking a specific role in regulation of late-firing origins (and see Supplemental Discussion 3).

Strikingly, the scaling of the replication timing program was completely lost in $m r c 1$ cells: Although S phase was extended over twofold, replication origins fired at much the same time as they did in wild-type cells. Only a small fraction of origins, confined to the very end of S phase, were delayed. Thus, MRC1 is a key factor in the scaling of the replication timing program.

\section{Discussion}

Using an unbiased screen, we identified a total of 14 genes whose deletion causes a significant extension of $S$ phase. Our screen enabled high-resolution detection of the $S$ phase pattern, revealing two novel effects. First, mutants of DNA replication factors, most notably the nonessential subunits of DNA polymerase $\varepsilon$, displayed distinct defects that are consistent with their requirement during 
A

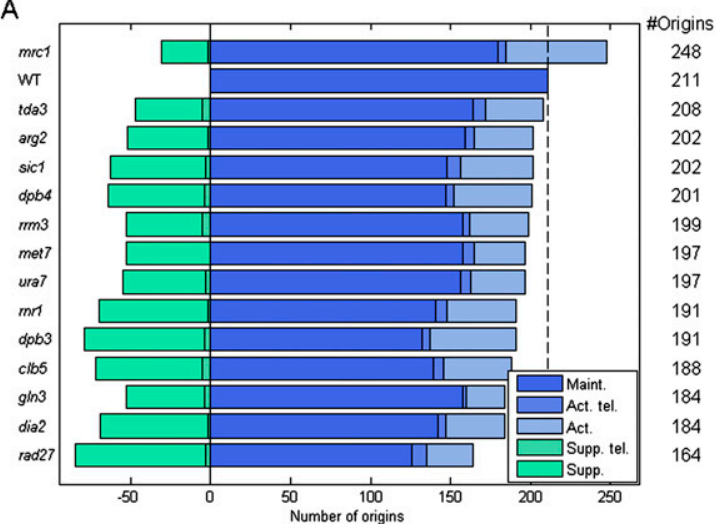

B

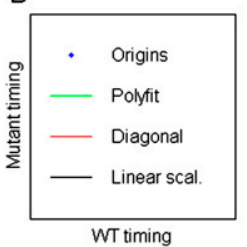

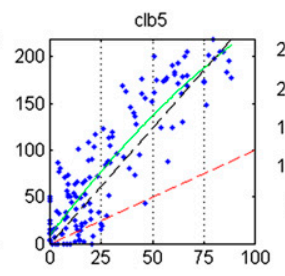

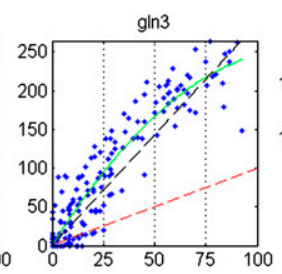

C

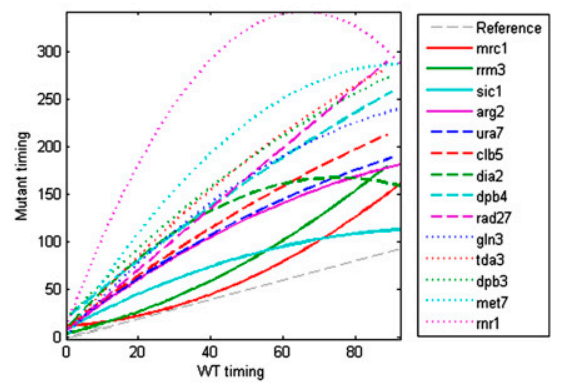

ura7

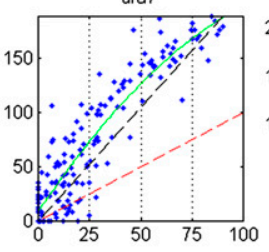

$\operatorname{mrc1}$

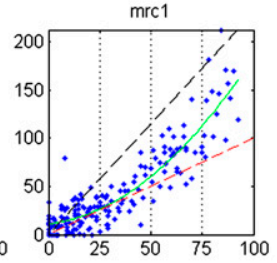

Figure 5. Scaling of the replication profiles. $(A)$ Number of replication origins in each mutant. The broken vertical line represents the number of origins active in the wild-type strain. Total active origins are further divided into origins that appear in both the wild type and mutant (maintained, "Maint.") and those that appear only in the mutant (activated, "Act."). This later group is further divided into telomeric origins ("Act. tel."; this includes the rDNA borders) and nontelomeric origins. Green bars represent origins that appear in the wild type but not in the mutants (suppressed, "Supp."), again divided into these found in telomeric ("Supp. tel.") vs. nontelomeric regions. (B) Scaling in all mutants apart from the mrc1 deletion strain: For each mutant, the scatter plot shows the activation timing of each maintained origin in the mutant ( $y$-axis) as a function of its activation time in the wild type ( $x$-axis). The wild-type data are scaled to $0-100$, and the mutant data are scaled according to the relative length of $S$ phase. Red broken lines represent identical timing between the wild type and mutant. The green line is a second-order polynomial fit to the data. The black broken line represents a perfect linear scaling. Typically, origin activation times are delayed more than by a linear factor. All mutants are shown in Supplemental Figure S5. (C) All polynomial fits (as in B) are shown together for comparison. The broken gray line represents no delay in origin activation. The differences between $\mathrm{mrc} 1$ and $r r m 3$ and sic1 are more pronounced than apparent from this comparative figure when taking into account the significantly longer $S$ phase of $m r c 1$ cells (see $B$ ).

specific S phase stages. Second, genetic interactions analysis of a gene group associated with dNTP synthesis suggested a key requirement for balanced nucleotide levels in S phase progression.

Perhaps the key result of our study is that the budding yeast replication timing program is remarkably robust (Fig. 6): Despite the varying $S$ phase durations and patterns, the temporal replication program remained largely the same in 13 of the 14 mutants identified in our screen. Most origins still fired, and their relative firing time was similar. In fact, firing of all origins was delayed relative to wild type, and the delay was largely proportional to the $S$ phase duration, maintaining a relatively fixed spacing of origins despite the slower replication kinetics. Similarly, we observed the same replication profile in wild-type cells grown under diverse environmental conditions that significantly alter transcriptional patterns (A Koren and N Barkai, unpubl.).

The only mutation that did not follow this pattern was mrc1. In these cells, origin firing time was not delayed, despite the approximately twofold increase in $\mathrm{S}$ phase duration. Moreover, a significant number of dormant origins were activated. These newly activated origins were distributed throughout $\mathrm{S}$ phase and might represent origins that are inactive in the wild-type background because of interference.

Mrc1 plays a dual role in the replication process, being important for replication fork stabilization, as well as a mediator of

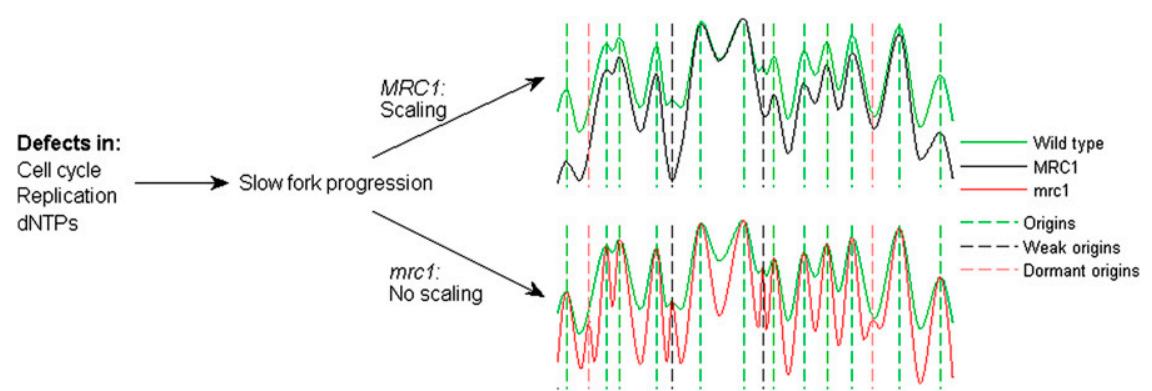

Figure 6. Model of replication timing control. Perturbations of normal replication fork progression (or origin activation), brought about by disruption of replication factors, cause a proportional delay in the activation timing of the different origins, maintaining their relative order of firing. While the structure of the replication timing program is overall maintained, certain origin are rendered inactive because of passive replication. Conversely, in mrc1-deleted cells with a similar disruption of replication, origins are not delayed and the overall structure of the replication program is altered. When combined with the slower progression of the replication fork, this enables the firing of dormant origins, which in wild type are passively replicated. The replication profiles (solid lines) correspond to: green, wild type (unperturbed; normal replication); black, cells subject to a replication perturbation (mutation) with intact MRC1 (scaled replication program); red, cells subject to a replication perturbation with defective MRC1 (no scaling). Broken lines correspond to different classes of replication origins: green, origins active in all situations; black, origins rendered inactive because of scaling when MRC1 is intact and replication is perturbed; red, normally inactive origins that become activated when replication is perturbed and MRC1 is nonfunctional.

\section{Genome Research www.genome.org}


the replication checkpoint. The possibility that scaling is mediated by the checkpoint response would be consistent with the observation that a mutation in the Rad53 checkpoint kinase enables the activation of numerous origins that are otherwise inhibited by HU treatment (Feng et al. 2006). Further studies will be required to address the role of the checkpoint pathway in scaling of the replication timing program.

\section{Methods}

\section{Strains and media}

All experiments were performed with the $S$. cerevisiae strains

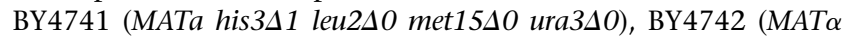

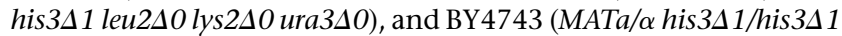

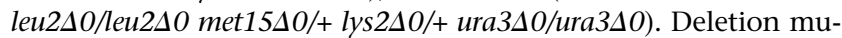
tants marked with the G418 resistance (KanMX) cassette (Giaever et al. 2002) were obtained from Euroscarf.

All deletion mutants used for microarray experiments and double mutant construction were verified to be correct clones by barcode-sequencing and were regenerated and tested by sporulating the corresponding heterozygote strains from the BY4743 background.

The medium used was YPD (1\% yeast extract, $2 \%$ peptone, $2 \%$ dextrose) with addition of Geneticin $(200 \mu \mathrm{g} / \mathrm{mL})(\mathrm{GIBCO})$ or nourseothricin (Nat) $(100 \mu \mathrm{g} / \mathrm{mL})$ (Werner Bioagents) when required. Cells were grown at $30^{\circ} \mathrm{C}$.

HU concentrations were $10-50 \mathrm{mM}$; higher concentrations resulted in extreme elongation of $S$ phase as well as cell swelling, the combination of which hindered FACS analysis. At the HU concentrations used, neither the wild type nor any of the mutants suffered any effect on viability (data not shown).

\section{Double mutant construction}

Deletion strains on the BY4741 background were mated with BY4742 to obtain heterozygous single mutant strains. These were transformed with plasmid p4339 (pCRII-TOPO::natRMX4; Tong et al. 2001) to convert them from G418-resistant to Nat-resistant. Random spore analysis was followed by selection for Nat+ and $M A T \alpha$. After FACS verification of phenotype detainment, these strains were backcrossed to the original strains (G418+ MATa). Double heterozygous strains were sporulated, and haploid double mutant clones were obtained by random spore analysis and selection for G418+ Nat+. All mutants were constructed in reciprocal crosses, and the profile from one of the crosses was chosen for presentation and further analysis. Mutants that were not obtained in reciprocal crosses were constructed again in each reciprocal cross. Mutants that did not have at least two reproducible profiles were removed from further analysis. This included the rnr1 sic1 strain and all combinations containing dia2, which had low reproducibility and several combinations of which were obtained despite being reported in the literature as synthetically lethal (Pan et al. 2006), suggesting that suppressor mutations are obtained in this background at high frequencies. The $m r c 1 \mathrm{rrm} 3$ double mutant was never obtained, consistent with its reported synthetic lethality (Torres et al. 2004; Szyjka et al. 2005). In addition, double mutant combinations that had $S$ phases shorter than both single mutants (i.e., suppressive interaction) were regenerated to verify that they are not the result of suppressor mutations.

\section{RT-PCR}

The primers used were CATTGTGGAAAACGAGCCAA and TGGC GTGTTGTTATTCTGGGA. The experiment was performed twice, in replicate samples, and results were averaged.

\section{FACS staining}

Logarithmic cultures were fixated with $70 \%$ ethanol. Fixated cells were washed twice with $50 \mathrm{mM}$ Tris- $\mathrm{HCl}(\mathrm{pH}$ 8), treated with $300 \mu \mathrm{L}$ of $1 \mathrm{mg} / \mathrm{mL}$ RNase A (Sigma) for $40 \mathrm{~min}$ at $37^{\circ} \mathrm{C}$, washed twice with Tris- $\mathrm{HCl}$, treated with $50 \mu \mathrm{L}$ of $20 \mathrm{mg} / \mathrm{mL}$ proteinase $\mathrm{K}$ (Sigma) for $1 \mathrm{~h}$ at $37^{\circ} \mathrm{C}$, washed once with Tris- $\mathrm{HCl}$, and incubated for $1 \mathrm{~h}$ in room temperature with $200 \mu \mathrm{L} \times 10$ (10-fold higher concentration than the reagent's working concentration recommended by the manufacturer) SYBR Green I (Invitrogen). Cells were sonicated for $5 \mathrm{sec}$, and $500 \mu \mathrm{L}$ of Tris- $\mathrm{HCl}$ was added.

\section{Genome-wide FACS screen}

Screening the entire yeast deletion collection was performed in 96-well plates using robotic DNA staining. The same protocol as that for single tubes was used with the following modifications: $200 \mu \mathrm{L}$ of cells were stained, RNaseA was diluted threefold and $200 \mu \mathrm{L}$ were used per reaction, proteinase $\mathrm{K}$ was diluted 20 -fold and $200 \mu \mathrm{L}$ were used per reaction, and SYBR Green was diluted 1:2.5 and $100 \mu \mathrm{L}$ were used per reaction. Tris- $\mathrm{HCl}$ was not added after sonication. Measurements were performed using the LSR-II HTS (high-throughput system), and 30,000 cells were analyzed.

Each mutant plate contained several empty wells, which served as controls for between-well carryover. In addition, two repeats of each of wild type, $d p b 3$ and rad27, were included in each plate as controls.

Strains with an S phase longer than wild type were reanalyzed in single tubes, and only those for which the long S phase was reproduced were analyzed further.

\section{FACS sorting}

The single-tube staining protocol was scaled up 10-fold, and no Tris-HCl was added after sonication. Sorting was performed with the Beckton-Dickinson FACSAria sorter at minimal flow rate and sorting speed of $\sim 20,000-30,000$ cells/sec. Two million cells were sorted from each of $G_{1}$ and $S$ phases. For the strains sic1, dia2, and $\mathrm{rad} 27, \mathrm{G}_{2}$ instead of $\mathrm{G}_{1}$ cells were used.

\section{FACS data analysis}

The locations of the $G_{1}$ and $G_{2}$ peaks were identified automatically by finding the maximal points in each half of the DNA content histograms. All $G_{1}$ and $G_{2}$ fits were scanned manually and corrected in cases where the automatic procedure misidentified the peaks (e.g., in cases where the histogram over $S$ phase is higher than that at the $G_{1}$ peak). Then, to remove cell doublets (i.e., gating), all cells with SYBR-Green width FACS readings above $5 \%$ of the 90th percentile that of the cells with DNA content between $10 \%$ above the $\mathrm{G}_{1}$ center and $10 \%$ below the $\mathrm{G}_{2}$ center ( $\mathrm{S}$ phase cells) were removed from further analysis. All profiles were aligned at the $G_{1}$ and $G_{2}$ peaks, and the distribution of cells in 200 bins was determined and normalized to the number of cells in the largest bin. Percentage of each cell cycle phase was determined by the area under the histogram with the borders between $G_{1}, S$, and $G_{2}$ phases determined manually.

Absolute length of the different cell cycle phases was obtained by multiplying the percentage of cells in each phase by the generation time of each strain. Generation time was determined by measuring optical density over an eight-hour time course starting from one hour after dilution of an overnight culture; this was repeated twice for each strain and averaged. 


\section{Double mutant interaction scores}

Expected double mutant phenotypes were predicted by adding the number of cells in each single mutant and subtracting the number of cells in the wild type (once), in each bin. Then, these in-silico predictions were subtracted from the actual measurements to obtain a vector the length of the bin number (i.e., 200) representing the genetic interaction at each point during the cell cycle. Positive values occur when the number of cells at any particular bin is higher than expected (corresponding to aggravating interactions) and negative when it is lower (alleviating interactions). Interaction scores were the maximal genetic interaction value in $\mathrm{S}$ phase when most values were positive and the minimal value when most values were negative. The double mutant matrix was clustered according to the correlations between the interaction scores.

\section{DNA preparation}

DNA from sorted cells was extracted with the MasterPure Yeast DNA purification kit (Epicentre) according to the manufacturer's instructions. Purified DNA was digested with 20 units of DpnII for $1 \mathrm{~h}$, cleaned with the Qiagen PCR purification kit, and eluted with $20 \mu \mathrm{L}$ of TE buffer. Four rounds of DNA amplification were performed using random octamers, Exo-Klenow polymerase (Invitrogen BioPrime ArrayCGH amplification kit), and dNTPs ( $2 \mathrm{mM}$ dATP, dCTP, dGTP; $0.8 \mathrm{mM}$ dTTP; $1.2 \mathrm{mM}$ amino-allyl-dUTP). The following reaction volumes were used: round 1: $20 \mu \mathrm{L}$ of DNA, $15 \mu \mathrm{L}$ of $\mathrm{H}_{2} \mathrm{O}, 5 \mu \mathrm{L}$ of primers, $8 \mu \mathrm{L}$ of dNTP mix, $2 \mu \mathrm{L}$ of Klenow. Round 2: $20 \mu \mathrm{L}$ of primers, $9 \mu \mathrm{L}$ of dNTP mix, and $1 \mu \mathrm{L}$ of Klenow were added to the round 1 reaction mixture. Round 3: $20 \mu \mathrm{L}$ of DNA, $4 \mu \mathrm{L}$ of $\mathrm{H}_{2} \mathrm{O}, 20 \mu \mathrm{L}$ of primers, $5 \mu \mathrm{L}$ of dNTP mix, $1 \mu \mathrm{L}$ of Klenow. Round $4: 4 \mu \mathrm{L}$ of $\mathrm{H}_{2} \mathrm{O}, 20 \mu \mathrm{L}$ of primers, $5 \mu \mathrm{L}$ of dNTP mix, and $1 \mu \mathrm{L}$ of Klenow were added to the round 3 reaction mixture. In all rounds, samples were boiled at $100^{\circ} \mathrm{C}$ for $5 \mathrm{~min}$ after addition of primers (and $\mathrm{H}_{2} \mathrm{O}$ in round 1) to the DNA, and the other reagents added afterward, on ice. Incubation for $4 \mathrm{~h}$ at $37^{\circ} \mathrm{C}$ was used in each round. After rounds 2 and 4, $5 \mu \mathrm{L}$ of stop buffer (BioPrime kit) was added to the reactions, and DNA was cleaned with the Qiagen PCR purification kit and eluted with $20 \mu \mathrm{L}$ at round 2 and $50 \mu \mathrm{L}$ at round 4 of TE buffer. Amplified DNA was ethanol-precipitated by adding $114 \mu \mathrm{L}$ of $100 \%$ ethanol, $5 \mu \mathrm{L}$ of $3 \mathrm{M} \mathrm{NaAc}$, and $1 \mu \mathrm{L}$ of glycogen and stored at $-20^{\circ} \mathrm{C}$.

\section{Labeling and hybridization}

Ethanol-precipitated DNA was washed three times for $10 \mathrm{~min}$ at $4{ }^{\circ} \mathrm{C}$ with $70 \%$ cold ethanol. Remaining ethanol was removed with a speedvac, and DNA was resuspended with $10 \mu \mathrm{L}$ of sodium bicarbonate buffer for $10 \mathrm{~min}$ at $37^{\circ} \mathrm{C}$. Cy3 and Cy5 were added to the $G_{1}$ (or $G_{2}$ ) and $S$ DNA samples and incubated in room temperature for $1.5 \mathrm{~h}$. Dye-swaps were used for repeat experiments. DNA was cleaned from free dye with the Strataprep kit (Stratagene). $\mathrm{G}_{1}$ (or $\mathrm{G}_{2}$ ) and $\mathrm{S}$ phase DNA, differentially labeled, were mixed and concentrated to $37.5 \mu \mathrm{L}$ with a Microcon column. Microarray hybridization was performed according to Agilent's instructions. Arrays used were Agilent yeast CGH $44 \times 4$ arrays. Arrays were scanned using the Agilent microarray scanner and features were extracted with the SpotReader software (Niles Scientific).

The data have been submitted to the Gene Expression Omnibus (GEO) under accession number GSE17120.

\section{Microarray data normalization}

Probes that were flagged by the feature extraction software or manually, had saturated intensity levels, or had lower intensities than the average +2 standard deviations of the negative control probes were removed from further analysis. Differences between the dyes were normalized by fitting a lowess curve to the data points ratio of $\mathrm{Cy} 3 / \mathrm{Cy} 5$ as a function of geometric mean intensity with a span of $10 \%$ of the number of points and subtracting the curve coordinates from the corresponding data points at each intensity.

Data were then organized by chromosomal coordinate.

\section{Data averaging and smoothing}

Each experiment was normalized to mean 0 and std 1, and the four experimental repeats of each strain are averaged using Tukey's biweight method (Hubbell et al. 2002) on three consecutive probes from each of the four repeats. The data were then linearly interpolated to have values equally spaced every $10 \mathrm{bp}$ from the leftmost to the rightmost probe on each chromosome.

The averaged data (as well as separate repeats) were smoothed using a smoothing spline (as implicated in the Matlab function Csaps), which optimizes the degree of curvature versus deviance from the data, with the parameter chosen determining the weight of these two criteria. The parameters chosen for the different chromosomes were a function of the chromosome length, as follows (numbers are the $-\log _{10}$ of the parameters provided to Csaps): chromosomes I, III, V, VI, VIII, IX: 13.25; chromosomes X, XI, XIV: 13.5; chromosomes II, XII, XIII, XVI: 13.75; chromosomes IV, VII, $\mathrm{XV}: 14$.

Spline-smoothing performs similarly to Fourier-convolution smoothing (Raghuraman et al. 2001) but does not have the caveat of causing telomeres (as well as borders of introduced gaps in the data such as the rDNA locus) to receive identical values (data not shown).

Chromosome XII, which includes the rDNA locus, was smoothed as two separate chromosomes, one on each side of the rDNA locus, with the same smoothing parameter.

Each chromosome was scaled to 0-100 (early to late replication, respectively). This was done chromosome-wise to prevent noise from particular chromosomes from affecting the entire data. All conclusions of the study remained unaltered when scaling the entire genome as a single vector on a $0-100$ scale.

\section{Autocorrelation analysis}

Pearson correlation coefficients were determined for paired sets of probes from the first to the last $-x+1$ probe, and the $x$-probe to the last probe, with $x$ varying between 1 and the length of the probe set. Autocorrelations are computed on the data as one continuous vector representing the whole genome.

\section{Origin assignments}

To compare origins between experiments, all the peaks in all averaged experiment sets, and separately all the peaks in all individual experiments, were clustered using hierarchical clustering with average linkage (Eisen et al. 1998). A distance cutoff of $14 \mathrm{~Kb}$ between cluster centers was used to determine separate origins. Peaks that did not cluster together in at least three of four repeats of any single strain were omitted from further analyses. The remaining peaks were used to define origins as the average peak locations in each cluster. In cases in which two peaks from the same strain belong to the same cluster, the one closest to the average position of all the rest of the peaks is maintained while the other is removed. The resultant origin cluster list is used to compare common origins between experiments. 
Subsequently, all assigned origins were compared to lists of verified origins (Nieduszynski et al. 2006), proARSs (Wyrick et al. 2001), likely origins (Nieduszynski et al. 2006), and nimARSs (Xu et al. 2006). An assigned origin was regarded as a known origin if it fell within $14 \mathrm{~kb}$ from the origins in any of these lists and was assigned to the first list in the above order that complied with this criterion, unless a subsequent list contained an origin closer by more than $5 \mathrm{~kb}$ to the tested origin. Origins that were farther than $14 \mathrm{~kb}$ from the origins in any of these lists were regarded as novel identified origins if, in addition, their minimal fork size was $>3 \%$ (on a $0 \%-100 \%$ scale) in either $m r c 1$ (in which origin locations are relatively more precise) or at least two other strains besides $c l b 5$, $d p b 3, d p b 4$, and $\arg 2$ (in which origin locations are relatively less precise). These origins are detailed in Supplemental Table S4 but were not studied further. Other origins were regarded as dubious and removed from subsequent analyses.

Assignment of origins in published data was performed by comparing with the assigned origin list above by the same criteria, but without reclustering.

\section{Peak-origin consistency}

"Peaks" refer to generated data while "origins" refer to previously published chromosomal coordinates of replication origins. The distances and $P$ values presented (Supplemental Table S3) refer to comparisons with an origin list combining both sequenceidentified origins and those predicted from at least two microarray studies, as collated previously (Nieduszynski et al. 2006), while replication profiles (Figs. 3B, 4C,D) present only "verified," sequence-identified origins. For $P$-value calculations, all theoretically possible peak locations (every $10 \mathrm{bp}$ ) were used to calculate the mean and standard deviation of the distances to their nearest origin. According to the central limit theorem, a subset of size $n$ of all possible peak locations would by chance have the same average distance and $1 / \sqrt{n}$ the standard deviation of all possible location distance distributions. This in turn is used to calculate the $Z$-score for the observed average distance between the tested peak list and the origin list to which it is compared. Notably, our distance calculations are an underestimate of the accuracy of the data, since not all active origins are represented in the external data set used for comparison.

\section{Clustering of correlation matrices}

Pearson correlation coefficients were applied to the complete data or origin data, calculated for all pairwise strain combinations. The resulting correlation coefficient matrix was clustered using hierarchical clustering (Eisen et al. 1998) with Euclidean distance, average linkage, and optimal leaf ordering (Bar-Joseph et al. 2001).

\section{Acknowledgments}

We thank Judith Berman, Martin Kupiec, Duncan Clarke, and members of our laboratory for helpful suggestions and comments on the manuscript, and Martin Kupiec and Stephen Elledge for yeast strains. This work was supported by the ERC and by the Hellen and Martin Kimmel award for innovative investigations.

\section{References}

Aladjem MI. 2007. Replication in context: Dynamic regulation of DNA replication patterns in metazoans. Nat Rev Genet 8: 588-600.

Alvino GM, Collingwood D, Murphy JM, Delrow J, Brewer BJ, Raghuraman MK. 2007. Replication in hydroxyurea: It's a matter of time. Mol Cell Biol 27: 6396-6406.
Araki H, Hamatake RK, Morrison A, Johnson AL, Johnston LH, Sugino A. 1991. Cloning DPB3, the gene encoding the third subunit of DNA polymerase II of Saccharomyces cerevisiae. Nucleic Acids Res 19: 48674872 .

Bar-Joseph Z, Gifford DK, Jaakkola TS. 2001. Fast optimal leaf ordering for hierarchical clustering. Bioinformatics 17: S22-S29.

Bell SP, Dutta A. 2002. DNA replication in eukaryotic cells. Annu Rev Biochem 71: $333-374$.

Blinder D, Magasanik B. 1995. Recognition of nitrogen-responsive upstream activation sequences of Saccharomyces cerevisiae by the product of the GLN3 gene. J Bacteriol 177: 4190-4193.

Cherest H, Thomas D, Surdin-Kerjan Y. 2000. Polyglutamylation of folate coenzymes is necessary for methionine biosynthesis and maintenance of intact mitochondrial genome in Saccharomyces cerevisiae. J Biol Chem 275: 14056-14063.

Donaldson AD, Raghuraman MK, Friedman KL, Cross FR, Brewer BJ, Fangman WL. 1998. CLB5-dependent activation of late replication origins in S. cerevisiae. Mol Cell 2: 173-182.

Eisen MB, Spellman PT, Brown PO, Botstein D. 1998. Cluster analysis and display of genome-wide expression patterns. Proc Natl Acad Sci 95: 14863-14868.

Feng W, Collingwood D, Boeck ME, Fox LA, Alvino GM, Fangman WL, Raghuraman MK, Brewer BJ. 2006. Genomic mapping of single-stranded DNA in hydroxyurea-challenged yeasts identifies origins of replication. Nat Cell Biol 8: 148-155.

Giaever G, Chu AM, Ni L, Connelly C, Riles L, Veronneau S, Dow S, LucauDanila A, Anderson K, Andre B, et al. 2002. Functional profiling of the Saccharomyces cerevisiae genome. Science 418: 387-391.

Green BM, Morreale RJ, Ozaydin B, Derisi JL, Li JJ. 2006. Genome-wide mapping of DNA synthesis in Saccharomyces cerevisiae reveals that mechanisms preventing reinitiation of DNA replication are not redundant. Mol Biol Cell 17: 2401-2414.

Hiratani IT, Ryba M, Itoh T, Yokochi M, Schwaiger CW, Chang Y, Lyou TM, Townes D, Schübeler D, Gilbert DM. 2008. Global reorganization of replication domains during embryonic stem cell differentiation. PLoS Biol 6: e245. doi: 10.1371/journal.pbio.0060245.

Huang M, Zhou Z, Elledge SJ. 1998. The DNA replication and damage checkpoint pathways induce transcription by inhibition of the Crt1 repressor. Cell 94: 595-605.

Hubbell E, Liu W-M, Mei R. 2002. Robust estimators for expression analysis. Bioinformatics 18: 1585-1592.

Ivessa AS, Lenzmeier BA, Bessler JB, Goudsouzian LK, Schnakenberg SL, Zakian VA. 2003. The Saccharomyces cerevisiae helicase Rrm3p facilitates replication past nonhistone protein-DNA complexes. Mol Cell 12: $1525-1536$.

Katou Y, Kanoh Y, Bando M, Noguchi H, Tanaka H, Ashikari T, Sugimoto K, Shirahige K. 2003. S-phase checkpoint proteins Tof1 and Mrc1 form a stable replication-pausing complex. Nature 424: 1078-1083.

Knott SR, Viggiani CJ, Tavare S, Aparicio OM. 2009. Genome-wide replication profiles indicate an expansive role for $\mathrm{Rpd} 3 \mathrm{~L}$ in regulating replication initiation timing or efficiency, and reveal genomic loci of Rpd3 function in Saccharomyces cerevisiae. Genes \& Dev 23: 10771090.

Koepp DM, Kile AC, Swaminathan S, Rodriguez-Rivera V. 2006. The F-box protein Dia2 regulates DNA replication. Mol Biol Cell 17: 1540-1548.

Lengronne A, Schwob E. 2002. The yeast CDK inhibitor Sic1 prevents genomic instability by promoting replication origin licensing in late $\mathrm{G}_{1}$. Mol Cell 9: 1067-1078.

McCune HJ, Danielson LS, Alvino GM, Collingwood D, Delrow JJ, Fangman WL, Brewer BJ, Raghuraman MK. 2008. The temporal program of chromosome replication: Genomewide replication in clb5 $\Delta$ Saccharomyces cerevisiae. Genetics 180: 1833-1847.

Morohashi H, Maculins T, Labib K. 2009. The amino-terminal TPR domain of Dia2 tethers SCF ${ }^{\text {Dia2 }}$ to the replisome progression complex. Curr Biol 19: $1943-1949$.

Murray AW. 2004. Recycling the cell cycle: Cyclins revisited. Cell 116: 221234.

Nieduszynski CA, Knox Y, Donaldson AD. 2006. Genome-wide identification of replication origins in yeast by comparative genomics. Genes \& Dev 20: $1874-1879$.

Nordlund P, Reichard P. 2006. Ribonucleotide reductases. Annu Rev Biochem 75: $681-706$.

Ohya T, Maki S, Kawasaki Y, Sugino A. 2000. Structure and function of the fourth subunit (Dpb4p) of DNA polymerase epsilon in Saccharomyces cerevisiae. Nucleic Acids Res 28: 3846-3852.

Osborn AJ, Elledge SJ. 2003. Mrc1 is a replication fork component whose phosphorylation in response to DNA replication stress activates Rad53. Genes \& Dev 17: 1755-1767.

Ozier-Kalogeropoulos O, Adeline MT, Yang WL, Carman GM, Lacroute F. 1994 Use of synthetic lethal mutants to clone and characterize a novel CTP synthetase gene in Saccharomyces cerevisiae. Mol Gen Genet 242: 431-439. 
Pan X, Ye P, Yuan DS, Wang X, Bader JS, Boeke JD. 2006. A DNA integrity network in the yeast Saccharomyces cerevisiae. Cell 124: 1069-1081.

Raghuraman MK, Winzeler EA, Collingwood D, Hunt S, Wodicka L, Conway A, Lockhart DJ, Davis RW, Brewer BJ, Fangman WL. 2001. Replication dynamics of the yeast genome. Science 294: 115-121.

Santocanale C, Diffley JF. 1998. A Mec1- and Rad53-dependent checkpoint controls late-firing origins of DNA replication. Nature 395: 615-618.

Schubeler D, Scalzo D, Kooperberg C, van Steensel B, Delrow J, Groudine M. 2002. Genome-wide DNA replication profile for Drosophila melanogaster. A link between transcription and replication timing. Nat Genet 32: 438442 .

Schwaiger M, Stadler MB, Bell O, Kohler H, Oakeley EJ, Schubeler D. 2009. Chromatin state marks cell-type- and gender-specific replication of the Drosophila genome. Genes \& Dev 23: 589-601.

Sclafani RA, Holzen TM. 2007. Cell cycle regulation of DNA replication. Annu Rev Genet 41: 237-280.

Shirahige K, Hori Y, Shiraishi K, Yamashita M, Takahashi K, Obuse C, Tsurimoto T, Yoshikawa H. 1998. Regulation of DNA-replication origins during cell-cycle progression. Nature 395: 618-621.

Szyjka SJ, Viggiani CJ, Aparicio OM. 2005. Mrc1 is required for normal progression of replication forks throughout chromatin in S. cerevisiae. Mol Cell 19: 691-697.

Tanaka S, Tak YS, Araki H. 2007. The role of CDK in the initiation step of DNA replication in eukaryotes. Cell Div 2: 16

Tong AHY, Evangelista M, Parsons AB, Xu H, Bader GD, Page N, Robinson M, Raghibizadeh S, Hogue CWV, Bussey H, et al. 2001. Systematic genetic analysis with ordered arrays of yeast deletion mutants. Science 294: 2364-2368.

Torres JZ, Schnakenberg SL, Zakian VA. 2004. Saccharomyces cerevisiae Rrm3p DNA helicase promotes genome integrity by preventing replication fork stalling: Viability of $r r m 3$ cells requires the intra-S-phase checkpoint and fork restart activities. Mol Cell Biol 24: 3198-3212.

Tourriere H, Versini G, Cordon-Preciado V, Alabert C, Pasero P. 2005. Mrc1 and Tof 1 promote replication fork progression and recovery independently of Rad53. Mol Cell 19: 699-706.

Verma R, Annan RS, Huddleston MJ, Carr SA, Reynard G, Deshaies RJ. 1997. Phosphorylation of Sic1p by G1 Cdk required for its degradation and entry into S phase. Science 278: 455-460.

Wyrick JJ, Aparicio JG, Chen T, Barnett JD, Jennings EG, Young RA, Bell SP Aparicio OM. 2001. Genome-wide distribution of ORC and MCM proteins in S. cerevisiae: High-resolution mapping of replication origins. Science 294: 2357-2360.

Xu W, Aparicio JG, Aparicio OM, Tavare S. 2006. Genome-wide mapping of ORC and Mcm2p binding sites on tiling arrays and identification of essential ARS consensus sequences in S. cerevisiae. BMC Genomics 7: 276. doi: 10.1186/1471-2164-7-276.

Yabuki N, Terashima H, Kitada K. 2002. Mapping of early firing origins on a replication profile of budding yeast. Genes Cells 7: 781-789.

Zegerman P, Diffley JF. 2007. Phosphorylation of Sld2 and Sld3 by cyclindependent kinases promotes DNA replication in budding yeast. Nature 445: 281-285.

Zhao X, Muller EG, Rothstein R. 1998. A suppressor of two essential checkpoint genes identifies a novel protein that negatively affects dNTP pools. Mol Cell 2: 329-340.

Zhao X, Chabes A, Domkin V, Thelander L, Rothstein R. 2001. The ribonucleotide reductase inhibitor Sml1 is a new target of the Mec1/ Rad53 kinase cascade during growth and in response to DNA damage. EMBO J 20: 3544-3553.

Received November 2, 2009; accepted in revised form March 3, 2010. 


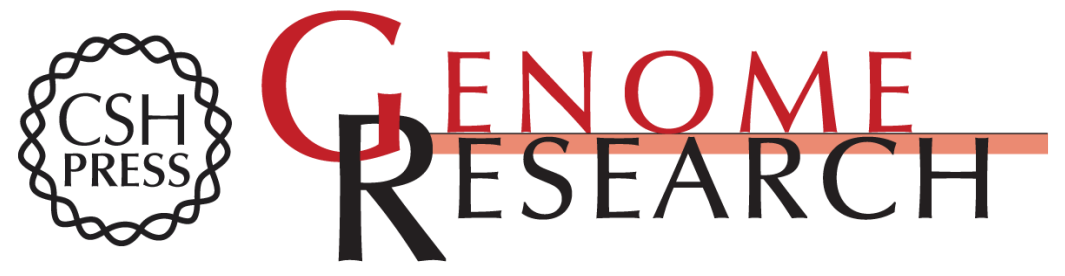

\section{MRC1-dependent scaling of the budding yeast DNA replication timing program}

Amnon Koren, llya Soifer and Naama Barkai

Genome Res. 2010 20: 781-790 originally published online March 10, 2010

Access the most recent version at doi:10.1101/gr.102764.109

Supplemental Material

References

License

Email Alerting Service
http://genome.cshlp.org/content/suppl/2010/03/11/gr.102764.109.DC1

This article cites 47 articles, 16 of which can be accessed free at: http://genome.cshlp.org/content/20/6/781.full.html\#ref-list-1

Receive free email alerts when new articles cite this article - sign up in the box at the top right corner of the article or click here.

\section{Affordable, Accurate Sequencing.}

To subscribe to Genome Research go to:

https://genome.cshlp.org/subscriptions 